\title{
Eigenvalues variations for Aharonov-Bohm operators
}

\author{
Corentin Léna
}

March 17, 2014

\begin{abstract}
We study how the eigenvalues of a magnetic Schrödinger operator of Aharonov-Bohm type depend on the singularities of its magnetic potential. We consider a magnetic potential defined everywhere in $\mathbb{R}^{2}$ except at a finite number of singularities, so that the associated magnetic field is zero. On a fixed planar domain, we define the corresponding magnetic Hamiltonian with Dirichlet boundary conditions, and study its eigenvalues as functions of the singularities. We prove that these functions are continuous, and in some cases even analytic. We sketch the connection of this eigenvalue problem to the problem of finding spectral minimal partitions of the domain.
\end{abstract}

\section{Introduction}

Aharonov-Bohm operators have been introduced in [1] as models of Schrödinger operators with a localized magnetic field. In addition to their physical relevance, it has been shown in 8 that these operators appear in the theory of spectral minimal partitions (see [9] for a definition of the latter object). In [4, 3, 5], the eigenvalues and eigenfunctions of an Aharonov-Bohm operator with Dirichlet boundary condition have thus been studied numerically to find minimal partitions. One of the aim of the present work is to support and generalize some observations made in these papers.

We define Aharonov-Bohm operators as follows. Let $\omega$ be an open and connected set in $\mathbb{R}^{2}$. As usual, we denote by $C_{c}^{\infty}(\omega)$ the set of smooth functions compactly supported in $\omega$. Generally speaking, let us consider a magnetic potential, that is to say a vector field $\mathbf{A} \in C^{\infty}\left(\omega, \mathbb{R}^{2}\right)$. We define the sesquilinear form $s_{\mathbf{A}}$ by

$$
s_{\mathbf{A}}(u, v)=\int_{\omega}(-i \nabla-\mathbf{A}) u \cdot \overline{(-i \nabla-\mathbf{A}) v} d x
$$

the quadratic form $q_{\mathbf{A}}$ by

$$
q_{\mathbf{A}}(u)=s_{\mathbf{A}}(u, u)
$$

and the norm $\|\cdot\|_{\mathbf{A}}$ by

$$
\|u\|_{\mathbf{A}}^{2}=\|u\|_{L^{2}(\omega)}^{2}+q_{\mathbf{A}}(u)
$$

for $u$ and $v$ in $C_{c}^{\infty}(\omega)$. 
The form domain $\mathcal{Q}_{\mathbf{A}}$ associated with $\mathbf{A}$ is defined as the completion of $C_{c}^{\infty}(\omega)$ under $\|\cdot\|_{\mathbf{A}}$. According to Friedrichs' Theorem, there exists a unique self-adjoint extension $-\Delta_{\mathbf{A}}$ of the densely defined, symmetric, and positive differential operator $(-i \nabla-\mathbf{A})^{2}$ acting on $C_{c}^{\infty}(\omega)$ whose domain $\mathcal{D}_{\mathbf{A}}$ is contained in $\mathcal{Q}_{\mathbf{A}}$ (see e.g. [16]). We call it the magnetic Hamiltonian on $\omega$ associated with A (note that we are imposing a Dirichlet boundary condition).

In the following, we denote by $\Omega$ an open, bounded, and connected set in $\mathbb{R}^{2}$ with a Lipschitz boundary $\partial \Omega$. For $v=\left(v_{1}, v_{2}\right) \in \mathbb{R}^{2}$, we note $v^{\perp}=\left(-v_{2}, v_{1}\right)$. For $X \in \mathbb{R}^{2}, \alpha \in \mathbb{R}$, and $x=\left(x_{1}, x_{2}\right) \in \mathbb{R}^{2} \backslash\{X\}$, we define

$$
\mathbf{A}_{\alpha}^{X}(x)=\frac{\alpha}{|x-X|^{2}}(x-X)^{\perp},
$$

and note $\mathbf{A}_{\alpha}^{X}(x)=\left(A_{\alpha, 1}^{X}(x), A_{\alpha, 2}^{X}(x)\right)$. In the rest of the paper, $N$ is an integer, $\mathbf{X}=\left(X_{1}, \ldots, X_{N}\right)$ an $N$-tuple of points in $\mathbb{R}^{2}$, with $X_{i} \neq X_{j}$ for $i \neq j$ (at least for now), and $\boldsymbol{\alpha}=\left(\alpha_{1}, \ldots \alpha_{N}\right) \in \mathbb{R}^{N}$. Let us denote by $\Omega_{\mathbf{X}}$ the open set $\Omega \backslash\left\{X_{1}, \ldots, X_{N}\right\}$. We define the Aharonov-Bohm potential associated with $\mathbf{X}$ and $\boldsymbol{\alpha}$ as the vector field

$$
\mathbf{A}_{\boldsymbol{\alpha}}^{\mathbf{X}}=\sum_{i=1}^{N} \mathbf{A}_{\alpha_{i}}^{X_{i}}
$$

Let us point out that the $X_{i}$ 's can be in $\mathbb{R}^{2} \backslash \Omega$, and in particular in $\partial \Omega$. The Aharonov-Bohm operator associated with $\mathbf{X}$ and $\boldsymbol{\alpha}$ is the magnetic Hamiltonian $-\Delta_{\mathbf{A}_{\alpha}^{\mathbf{x}}}$ on $\Omega_{\mathbf{X}}$. We denote it by $-\Delta_{\boldsymbol{\alpha}}^{\mathbf{X}}$, and the associated form domain and quadratic form by $\mathcal{Q}_{\boldsymbol{\alpha}}^{\mathbf{X}}$ and $q_{\boldsymbol{\alpha}}^{\mathbf{X}}$ respectively. Along the paper, we make frequent references to the Dirichlet realization of the Laplacian on $\Omega$, and to the sequence of its eigenvalues. We denote them by $-\Delta_{\Omega}^{D}$ and $\left(\lambda_{k}^{D}(\Omega)\right)_{k \geq 1}$ respectively.

Let us conclude by a few remarks of a more physical nature. To an AharonovBohm potential is associated a magnetic field

$$
\mathbf{B}=\operatorname{Curl} \mathbf{A}_{\boldsymbol{\alpha}}^{\mathbf{X}}=\partial_{x_{1}} A_{\alpha, 2}^{X}-\partial_{x_{2}} A_{\alpha, 1}^{X}=\sum_{i=1}^{N} \alpha_{i} \delta_{X_{i}},
$$

which is a measure. If $B_{i}$ is a small disk centered at $X_{i}$, such that $X_{j} \notin B_{i}$ for $j \neq i$,

$$
\alpha_{i}=\frac{1}{2 \pi} \int_{B_{i}} \mathbf{B} .
$$

The coefficient $\alpha_{i}$ can therefore be called the normalized (magnetic) flux at $X_{i}$. Let finally note that for any closed loop $\gamma$ in $\mathbb{R}^{2} \backslash \cup_{i=1}^{N}\left\{X_{i}\right\}$,

$$
\oint_{\gamma} \mathbf{A}_{\boldsymbol{\alpha}}^{\mathbf{X}}(s) d \mathbf{s}=2 \pi \sum_{i=1}^{N} \operatorname{ind}_{\gamma}\left(X_{i}\right) \alpha_{i}
$$

where $\operatorname{ind}_{\gamma}\left(X_{i}\right)$ is the winding number of $\gamma$ around $X_{i}$.

We use in this paper the following characterization of the form domain $\mathcal{Q}_{\boldsymbol{\alpha}}^{\mathbf{X}}$, which follows from Hardy-type inequalities proved in [12, 2, 14].

Proposition 1.1. Let $\mathcal{H}_{\boldsymbol{\alpha}}^{\mathbf{X}}$ be defined as

$$
\mathcal{H}_{\boldsymbol{\alpha}}^{\mathbf{X}}=\left\{u \in L^{2}(\Omega) ;\left(-i \nabla-\mathbf{A}_{\boldsymbol{\alpha}}^{\mathbf{X}}\right) u \in L^{2}(\Omega)\right\}
$$


with the natural scalar product. Then $\mathcal{H}_{\boldsymbol{\alpha}}^{\mathbf{X}}$ is a Hilbert space compactly embedded in $L^{2}(\Omega)$. Furthermore, there exists a continuous mapping $\gamma_{0}: \mathcal{H}_{\boldsymbol{\alpha}}^{\mathbf{X}} \rightarrow L_{l o c}^{2}(\partial \Omega)$ such that for any $u \in C_{c}^{\infty}\left(\bar{\Omega} \backslash\left\{X_{1}, \ldots, X_{N}\right\}\right), \gamma_{0} u=u_{\mid \partial \Omega}$. A function $u$ in $L^{2}(\Omega)$ belongs to $\mathcal{Q}_{\boldsymbol{\alpha}}^{\mathbf{X}}$ if, and only if, $u \in \mathcal{H}_{\boldsymbol{\alpha}}^{\mathbf{X}}$ and $\gamma_{0} u=0$.

As a consequence of the compact embedding, the spectrum of $-\Delta_{\alpha}^{\mathbf{X}}$ consists in a sequence of real eigenvalues converging to $+\infty$. Let $\left(\lambda_{k}(\mathbf{X}, \boldsymbol{\alpha})\right)_{k \geq 1}$ be this sequence, the eigenvalues being arranged in non-decreasing order and counted with multiplicity. We are interested in the functions $\mathbf{X} \mapsto \lambda_{k}(\mathbf{X}, \boldsymbol{\alpha})$. Let us note that according to our definition of $\mathbf{A}_{\boldsymbol{\alpha}}^{\mathbf{X}}, \lambda_{k}(\mathbf{X}, \boldsymbol{\alpha})$ is defined only for $\mathbf{X} \in \mathbb{R}^{2 N}$ such that $X_{i} \neq X_{j}$ for $i \neq j$. To state the following result, it is convenient to extend the function $\mathbf{X} \mapsto \lambda_{k}(\mathbf{X}, \boldsymbol{\alpha})$ to $\mathbb{R}^{2 N}$. We define $\lambda_{k}(\mathbf{X}, \boldsymbol{\alpha})$ as the $k$-th eigenvalue of the operator $-\Delta_{\boldsymbol{\beta}}^{\mathbf{Y}}$, where the $M$-tuple $\mathbf{Y}=\left(Y_{1}, \ldots, Y_{M}\right)$ contains once, and only once, each point appearing in $\mathbf{X}=\left(X_{1}, \ldots, X_{N}\right)$ and where $\boldsymbol{\beta}=\left(\beta_{1}, \ldots, \beta_{M}\right)$ with

$$
\beta_{j}=\sum_{i, X_{i}=Y_{j}} \alpha_{i}, \forall 1 \leq j \leq M .
$$

For instance, with this definition, $\lambda_{k}\left(\left(X_{1}, X_{2}, X_{3}\right),\left(\alpha_{1}, \alpha_{2}, \alpha_{3}\right)\right)$, with $X_{1}=X_{2}$ and $X_{1} \neq X_{3}$, is $\lambda_{k}\left(\left(X_{1}, X_{3}\right),\left(\alpha_{1}+\alpha_{2}, \alpha_{3}\right)\right)$.

Theorem 1.2. For any given $k \geq 1$ and $\boldsymbol{\alpha} \in \mathbb{R}^{N}$, the function $\mathbf{X} \mapsto \lambda_{k}(\mathbf{X}, \boldsymbol{\alpha})$ is continuous in $\mathbb{R}^{2 N}$.

Let us note that this result has more implications than it may appear at first glance. In particular, it implies continuity of the eigenvalues in the case of one point tending to the boundary of $\Omega$, or in the case of coalescing points.

Additional regularity is easily obtained when the poles are distinct and far from the boundary. We prove the following result.

Theorem 1.3. Let $\mathbf{e}_{1}=(1,0)$ and $\mathbf{e}_{2}=(0,1)$ be the vectors of the canonical basis of $\mathbb{R}^{2}$. Assume that $\mathbf{X} \in \mathbb{R}^{2 N}$ and $k \geq 1$ are such that $X_{i} \notin \partial \Omega$ for $1 \leq i \leq N, X_{i} \neq X_{j}$ for $i \neq j$, and $\lambda_{k}(\mathbf{X}, \boldsymbol{\alpha})$ is a simple eigenvalue. Then the function

$\left(t_{1}, t_{2}, \ldots, t_{2 N-1}, t_{2 N}\right) \mapsto \lambda_{k}\left(\left(X_{1}+t_{1} \mathbf{e}_{1}+t_{2} \mathbf{e}_{2}, \ldots, X_{N}+t_{2 N-1} \mathbf{e}_{1}+t_{2 N} \mathbf{e}_{2}\right), \boldsymbol{\alpha}\right)$

is analytic in a neighborhood of 0 in $\mathbb{R}^{2 N}$.

In general, an eigenfunction $u$ of the operator $-\Delta_{\alpha}^{\mathbf{X}}$ is complex-valued and the set $u^{-1}(\{0\})$ consists in isolated points of $\Omega$. However, in the case where $\alpha_{i} \in \mathbb{Z}+1 / 2$ for all $1 \leq i \leq N$, we can define a class of eigenfunctions for which the notion of nodal set is meaningful. To this end, as in [7, we define a suitable unitary antilinear operator $K_{\mathbf{X}}$ (see Section 4 ) and call $K_{\mathbf{X}}$-real a function $u$ such that $K_{\mathbf{X}} u=u$. If $u$ is a $K_{\mathbf{X}}$-real eigenfunction, the set $u^{-1}(\{0\})$ is locally a regular curve, except at the poles and maybe at a finite number of points where curves cross. By analogy with the terminology used for real eigenfunctions of the operator $-\Delta_{\Omega}^{D}$, we call $u^{-1}(\{0\})$ the nodal set and its component curves the nodal lines. Following a suggestion of Susanna Terracini, we have studied the derivatives of an eigenvalue with respect to a pole when at least three nodal lines of an associated $K_{\mathbf{X}}$-real eigenfunction meet at this pole. It has already 
been shown in [15] that if the function $Y \mapsto \lambda_{k}(Y, 1 / 2)$ has a critical point at $X$ and if $\lambda_{k}(X, 1 / 2)$ is simple, an associated $K_{\mathbf{X}}$-real eigenfunction $u$ has at least three nodal lines meeting at $X$. By modifying our proof of Theorem 1.3 and using results from [2] on the structure of the nodal set, we prove the converse.

Theorem 1.4. Let $\boldsymbol{\alpha} \in \mathbb{R}^{N}$ and $\mathbf{X} \in \mathbb{R}^{2 N}$. Let us fix $1 \leq i \leq N$ with $X_{i} \in \Omega$. For $\mathbf{v} \in \mathbb{R}^{2}, t \in \mathbb{R}$, and $k \geq 1$, we define

$$
\mathbf{X}(t)=\left(X_{1}, \ldots, X_{i}+t \mathbf{v}, \ldots, X_{N}\right)
$$

and $\lambda_{k}(t)=\lambda_{k}(\mathbf{X}(t), \boldsymbol{\alpha})$. Let us assume that $\lambda_{k}(0)$ is simple and has a $K_{\mathbf{X}}$-real eigenfunction $u$ with at least three nodal lines that meet at $X_{i}$. Then $\lambda_{k}^{\prime}(0)=0$.

During the writing of this work, S. Terracini showed us a preliminary version of the paper [6]. It contains a similar continuity result, restricted to the case of one pole and assuming $\Omega$ to be simply connected with a $C^{\infty}$-boundary. It also contains a stronger version of Theorem 1.4. The main contribution of our paper is in the generality of Theorem 1.2 , which allows us to treat coalescing poles, and in the use of the Kato-Rellich regular perturbation theory to give a simpler proof of Theorem 1.4 .

The paper is organized as follows. We first recall the definition of a gauge transformation of the magnetic potential, and the fact that it preserves the spectrum of the associated magnetic Hamiltonian. We then give a criterion for $-\Delta_{\boldsymbol{\alpha}}^{\mathbf{X}}$ to be unitarily equivalent to $-\Delta_{\Omega}^{D}$. We recall a Hardy-type inequality from [12, 2], some of its consequences, and a non-concentration inequality for functions in $\mathcal{Q}_{\boldsymbol{\alpha}}^{\mathbf{X}}$. We give some indications on the proof and the usefulness of Proposition 1.1. In a second part, we show the continuity of the eigenvalues with respect to the poles (Theorem 1.2). We then apply this theorem to some examples: we study the limits of the eigenvalues when the operator has a pole moving toward the boundary of the domain (Corollary 3.4) or two poles moving toward one another (Corollary 3.5). We show the analyticity of the eigenvalues with respect to the poles under some restrictive conditions (Theorem 1.3), and consider their critical points (Theorem 1.4). In the last section, we discuss the connection of these results with spectral minimal partitions.

I would like to thank my advisors, Virginie Bonnaillie-Noël and Bernard Helffer, for directing me toward this subject, and for many suggestions and corrections. Virginie Bonnaillie-Noël also sent me the numerical computations and pictures used in Section 5. I benefited greatly from exchanges with Susanna Terracini and Luc Hillairet. In particular, Lemma 2.7 and the method used to prove Theorem 1.2 are taken from unpublished notes kindly communicated by Luc Hillairet.

\section{Gauge invariance and form domain}

This section consists in a review of some useful results. We first recall how Aharonov-Bohm operators are modified under a gauge transformation. We only need a few classical properties of these transformations (see for instance [13]), that we state without proof. We recall a Hardy-type inequality taken from [12, 2. We prove that functions in the from domain satisfy a non-concentration property. Finally, we sketch the proof Proposition 1.1 and describe how it is used in proving the continuity of the eigenvalues. 


\subsection{Gauge transformation}

Definition 2.1. A gauge transformation acts on pairs vector field-function as $(\mathbf{A}, u) \mapsto\left(\mathbf{A}^{*}, u^{*}\right)$, with

$$
\left\{\begin{array}{l}
\mathbf{A}^{*}=\mathbf{A}-i \frac{\nabla \psi}{\psi}, \\
u^{*}=\psi u
\end{array}\right.
$$

where $\psi \in C^{\infty}(\omega, \mathbb{C})$ satisfies $|\psi|=1$. The function $\psi$ is called a gauge function on $\omega$. Two magnetic potentials are said to be gauge equivalent when the second one can be obtained from the first by a gauge transformation.

We see immediately from the definition that a gauge transformation does not change the magnetic field $\mathbf{B}=\operatorname{curl} \mathbf{A}$, nor the probability distribution $|u|^{2}$.

As a consequence of [13, Theorem 1.2], we also have the following result.

Proposition 2.2. If $\mathbf{A}$ and $\mathbf{A}^{*}$ are two gauge equivalent magnetic potentials in $C^{\infty}\left(\omega, \mathbb{R}^{2}\right)$, the operators $-\Delta_{\mathbf{A}^{*}}$ and $-\Delta_{\mathbf{A}}$ are unitarily equivalent.

Due to the possible multi connectedness of the open set $\omega$, the equation $\operatorname{curl} \mathbf{A}=0$ is a necessary but not sufficient condition for the magnetic potential A to be gauge equivalent to 0 . This is the basis of the so-called Aharonov-Bohm effect, studied in [1]. We now give a criterion for the gauge equivalence, taken from [7].

Lemma 2.3. Let $\mathbf{A} \in C^{\infty}\left(\omega, \mathbb{R}^{2}\right)$. It is gauge equivalent to 0 if, and only if,

$$
\frac{1}{2 \pi} \oint_{\gamma} \mathbf{A}_{\boldsymbol{\alpha}}^{\mathbf{X}}(s) d \mathbf{s}
$$

is an integer for any loop $\gamma$ contained in $\omega$.

\subsection{A Hardy-type inequality}

Let us now recall a useful result from [12, which we express in the formulation of [2].

Lemma 2.4. Let $X \in \mathbb{R}^{2}$ and $\alpha \in \mathbb{R} \backslash \mathbb{Z}$. For every $0 \leq \rho_{1}<\rho_{2}$ and $u \in C_{c}^{\infty}\left(\mathbb{R}^{2} \backslash\{X\}\right)$,

$$
\int_{\mathcal{C}\left(X, \rho_{1}, \rho_{2}\right)} \frac{|u|^{2}}{|x-X|^{2}} d x \leq \operatorname{dist}(\alpha, \mathbb{Z})^{-2} \int_{\mathcal{C}\left(X, \rho_{1}, \rho_{2}\right)}\left|\left(-i \nabla-\mathbf{A}_{\alpha}^{X}\right) u\right|^{2} d x,
$$

with

$$
\mathcal{C}\left(X, \rho_{1}, \rho_{2}\right)=\left\{x \in \mathbb{R}^{2} ; \rho_{1}<|x-X|<\rho_{2}\right\} .
$$

We can prove the preceding statement by using polar coordinates, expanding the integrand in Fourier series in the angular variable, and applying Parseval's formula. The details can be found in [2].

Corollary 2.5. If $\alpha_{i} \notin \mathbb{Z}$ for each $1 \leq i \leq N$, then $\mathcal{H}_{\boldsymbol{\alpha}}^{\mathbf{X}}$ is continuously embedded in $H^{1}(\Omega)$.

Proof. Let $u \in \mathcal{Q}_{\boldsymbol{\alpha}}^{\mathbf{X}}$. Far from the poles, $u$ is locally in $H^{1}$. In the neighborhood of one pole, we can bring the flux of all the other poles to zero thanks to a local gauge transformation, and prove that $u$ is locally in $H^{1}$ with the help of Inequality (2.2). Using these ideas, we obtain an explicit control on the $H^{1}$-norm of $u$ and thus prove the embedding. 


\subsection{A non-concentration inequality}

We now establish a non-concentration result. It is a slight variant of an inequality that was communicated to us by Luc Hillairet. A similar result is proved in [10, Lem. 3.2.]. Let us first give it for $H^{1}$-functions.

Lemma 2.6. For any $0<\nu<1$, there exists a constant $C_{\nu} \geq 0$ such that, for all $u \in H^{1}\left(\mathbb{R}^{2}\right), x_{0} \in \Omega$, and $r>0$,

$$
\|u\|_{L^{2}\left(B\left(x_{0}, r\right)\right)} \leq C_{\nu} r^{\nu}\|u\|_{H^{1}\left(\mathbb{R}^{2}\right)}
$$

where

$$
B\left(x_{0}, r\right)=\left\{x \in \mathbb{R}^{2} ;\left|x-x_{0}\right|<r\right\} .
$$

Proof. The proof is a direct application of the continuous embedding $H^{1}\left(\mathbb{R}^{2}\right) \subset$ $L^{p}\left(\mathbb{R}^{2}\right)$, valid for any $2 \leq p<\infty$. Let $u \in H^{1}\left(\mathbb{R}^{2}\right)$. According to the Hölder inequality,

$\int_{B\left(x_{0}, r\right)}|u|^{2} d x \leq\left|B\left(x_{0}, r\right)\right|^{1 / q}\left(\int_{B\left(x_{0}, r\right)}|u|^{2 q^{\prime}} d x\right)^{1 / q^{\prime}} \leq\left|B\left(x_{0}, r\right)\right|^{1 / q}\|u\|_{L^{2 q^{\prime}\left(\mathbb{R}^{2}\right)}}^{2}$

for any $q$ and $q^{\prime}$ greater than 1 such that $1 / q+1 / q^{\prime}=1$. We chose $q=1 / \nu$, and obtain

$$
\int_{B\left(x_{0}, r\right)}|u|^{2} d x \leq C_{1} r^{2 \nu}\|u\|_{L^{\frac{2}{1-\nu}}\left(\mathbb{R}^{2}\right)}^{2},
$$

where $C_{1}$ is a constant depending only on $\nu$. Since $H^{1}\left(\mathbb{R}^{2}\right)$ is continuously embedded in $L^{\frac{2}{1-\nu}}\left(\mathbb{R}^{2}\right)$, we finally get

$$
\int_{B\left(x_{0}, r\right)}|u|^{2} d x \leq C_{2} r^{2 \nu}\|u\|_{H^{1}\left(\mathbb{R}^{2}\right)}^{2},
$$

where $C_{2}$ is a constant depending only on $\nu$.

We now extend this result to functions in the form domain $\mathcal{Q}_{\alpha}^{\mathbf{X}}$ in the case $\nu=1 / 2$.

Lemma 2.7. There exists a constant $C \geq 0$ such that, for any $x_{0} \in \Omega, r>0$, and $u \in \mathcal{Q}_{\boldsymbol{\alpha}}^{\mathbf{X}}$,

$$
\|u\|_{L^{2}\left(B\left(x_{0}, r\right)\right)}^{2} \leq C r\left(\|u\|_{L^{2}\left(\mathbb{R}^{2}\right)}^{2}+\left\|\left(-i \nabla-\mathbf{A}_{\boldsymbol{\alpha}}^{\mathbf{X}}\right) u\right\|_{L^{2}\left(\mathbb{R}^{2}\right)}^{2}\right) .
$$

Proof. Let us first consider the case where $u \in C_{c}^{\infty}\left(\Omega_{\mathbf{X}}\right)$. Then $|u| \in H^{1}\left(\mathbb{R}^{2}\right)$ and inequality 2.3 yields

$$
\|u\|_{L^{2}\left(B\left(x_{0}, r\right)\right)}^{2} \leq C r\left(\|u\|_{L^{2}\left(\mathbb{R}^{2}\right)}^{2}+\|\nabla|u|\|_{L^{2}\left(\mathbb{R}^{2}\right)}^{2}\right)
$$

for some constant $C$ independent of $u$ and $r$. According to the diamagnetic inequality, $|\nabla| u|(x)| \leq\left|\left(-i \nabla-\mathbf{A}_{\boldsymbol{\alpha}}^{\mathbf{X}}\right) u(x)\right|$ for almost every $x \in \mathbb{R}^{2}$. We obtain

$$
\|u\|_{L^{2}\left(B\left(x_{0}, r\right)\right)}^{2} \leq C r\left(\|u\|_{L^{2}\left(\mathbb{R}^{2}\right)}^{2}+\left\|\left(-i \nabla-\mathbf{A}_{\boldsymbol{\alpha}}^{\mathbf{X}}\right) u\right\|_{L^{2}\left(\mathbb{R}^{2}\right)}^{2}\right) .
$$

Since $\mathcal{Q}_{\boldsymbol{\alpha}}^{\mathbf{X}}$ is defined as the closure of $C_{c}^{\infty}\left(\Omega_{\mathbf{X}}\right)$ for the norm $\|\cdot\|_{\mathbf{X}}^{\mathbf{\alpha}}$, the inequality still holds for functions in $\mathcal{Q}_{\boldsymbol{\alpha}}^{\mathrm{X}}$. 


\subsection{Characterization of the form domain}

Since the characterization of Proposition 1.1 is standard, we do not give the details of its proof. The reader can refer to [15, Lemma 2.1.] for the case of one pole with a non-integer flux. The poles having integer flux can be dealt with using a local gauge transformation, and the fact that a point has $H^{1}$-capacity zero in $\mathbb{R}^{2}$. The operator $\gamma_{0}$ appearing in the statement of Proposition 1.1 is simply the classical boundary trace operator, or possibly its conjugate by a gauge transformation.

Let us comment in more details on the condition $\left(-i \nabla-\mathbf{A}_{\boldsymbol{\alpha}}^{\mathbf{X}}\right) u \in L^{2}(\Omega)$ for a function $u \in \mathcal{Q}_{\boldsymbol{\alpha}}^{\mathbf{X}}$. Far from the poles, it simply means that $u$ is locally in $H^{1}$. In a neighborhood of a pole $X_{i}$, its meaning depends on the normalized flux $\alpha_{i}$. If $\alpha_{i} \notin \mathbb{Z}$, Inequality 2.2 shows that $u$ is locally in $H^{1}$ (as seen in the proof of Corollary 2.5), with the additional integrability condition $u /\left|x-X_{i}\right| \in L^{2}(\Omega)$. If $\alpha_{i} \in \mathbb{Z}$, the condition means that there is a local gauge transformation $\psi$ such that $u^{*}=\psi u$ is locally in $H^{1}$, but does not mean that $u$ is locally in $H^{1}$. Indeed this is not the case as soon as $\alpha_{i} \neq 0$, because then the function $(r, \theta) \mapsto e^{2 i \pi \alpha_{i} \theta}$ (expressed in polar coordinates) is not $H^{1}$ in a neighborhood of 0 .

Most of the quoted authors have restricted themselves to the case where no $\alpha_{i}$ is an integer, and the form domain is contained in $H^{1}(\Omega)$. This is for example the case in [2, 15, 6]. This does not introduce any restriction for fixed poles, since an integer flux can be brought to zero by a gauge transformation defined on $\Omega_{\mathbf{X}}$. However we want to prove in this paper, among other results, the continuity of the eigenvalues when several poles, each with a non-integer flux, coalesce into a single pole whose flux that can be an integer (see for instance Corollary 3.5. The formulation of Proposition 1.1. which does not distinguish between integer and non-integer fluxes, allows us to do this.

\section{Continuity with respect to the poles}

\subsection{Main statement}

Let us first restate Theorem 1.2

Proposition 3.1. Let $\left(\mathbf{X}^{n}\right)_{n \geq 1}$ be a sequence of points in $\mathbb{R}^{2 N}$ such that $\mathbf{X}^{n} \rightarrow$ $\mathbf{X}$ when $n \rightarrow+\infty$. Then, for each $k \geq 1, \lambda_{k}\left(\mathbf{X}^{n}, \boldsymbol{\alpha}\right) \rightarrow \lambda_{k}(\mathbf{X}, \boldsymbol{\alpha})$ as $n \rightarrow+\infty$.

The proof is based on two key lemmas which will be stated and proved in the next subsections.

\subsection{An extraction lemma}

The following lemma is the central part of the proof of Proposition 3.1. Its proof uses the weak compactness of the unit ball of $H^{1}$, the non-concentration result of Lemma 2.7, and the characterization of the form domain $\mathcal{Q}_{\boldsymbol{\alpha}}^{\mathbf{X}}$ given in Proposition 1.1

Lemma 3.2. Let $\left(\lambda_{n}\right)_{n \geq 1}$ be a sequence of eigenvalues associated with $-\Delta_{\boldsymbol{\alpha}}^{\mathbf{X}^{n}}$ and $\left(u_{n}\right)_{n \geq 1}$ a sequence of corresponding normalized eigenfunctions. Assume that $\lambda_{n} \rightarrow \lambda$ when $n \rightarrow+\infty$. Then there is a strictly increasing sequence of integers $\left(n_{p}\right)_{p \geq 1}$ and a function $u \in \mathcal{Q}_{\boldsymbol{\alpha}}^{\mathbf{X}}$ such that 
i) $u$ is an eigenfunction of $-\Delta_{\alpha}^{\mathbf{X}}$ associated with the eigenvalue $\lambda$,

ii) $u_{n_{p}} \rightarrow u$ when $p \rightarrow \infty$ in $L^{2}(\Omega)$ (strongly),

iii) $u_{n_{p}}(x) \rightarrow u(x)$ when $p \rightarrow \infty$ for almost every $x$ in $\Omega$.

Proof. For $m \geq 1$, we define $S_{m}=\cup_{i=1}^{N} \overline{B\left(X_{i}, \frac{1}{m}\right)}$ and $\Omega_{m}=\Omega \backslash S_{m}$. Let us fix $m \geq 1$. Then, for any integer $n$ large enough so that $\Omega_{m}$ does not contain any point of $\mathbf{X}^{n}$,

$$
\left\|u_{n}\right\|_{L^{2}\left(\Omega_{m}\right)} \leq 1
$$

and

$$
\begin{aligned}
& \left\|\left(-i \nabla-\mathbf{A}_{\boldsymbol{\alpha}}^{\mathbf{X}}\right) u_{n}\right\|_{L^{2}\left(\Omega_{m}\right)}^{2} \\
& \quad \leq 2\left\|\left(-i \nabla-i \mathbf{A}_{\boldsymbol{\alpha}}^{\left.\mathbf{X}^{n}\right)}\right) u_{n}\right\|_{L^{2}\left(\Omega_{m}\right)}^{2}+2\left\|\mathbf{A}_{\boldsymbol{\alpha}}^{\mathbf{X}^{n}}-\mathbf{A}_{\boldsymbol{\alpha}}^{\mathbf{X}}\right\|_{L^{\infty}\left(\Omega_{m}\right)}^{2}\left\|u_{n}\right\|_{L^{2}\left(\Omega_{m}\right)}^{2} .
\end{aligned}
$$

We have

$$
\begin{gathered}
\left\|\left(-i \nabla-\mathbf{A}_{\boldsymbol{\alpha}}^{\mathbf{X}^{n}}\right) u_{n}\right\|_{L^{2}\left(\Omega_{m}\right)}^{2} \leq\left\|\left(-i \nabla-\mathbf{A}_{\boldsymbol{\alpha}}^{\mathbf{X}^{n}}\right) u_{n}\right\|_{L^{2}(\Omega)}^{2}=\lambda_{n} \leq \sup _{q \geq 1} \lambda_{q}, \\
\lim _{n \rightarrow \infty}\left\|\mathbf{A}_{\boldsymbol{\alpha}}^{\mathbf{X}^{n}}-\mathbf{A}_{\boldsymbol{\alpha}}^{\mathbf{X}}\right\|_{L^{\infty}\left(\Omega_{m}\right)}=0
\end{gathered}
$$

and

$$
\left\|\mathbf{A}_{\boldsymbol{\alpha}}^{\mathbf{X}}\right\|_{L^{\infty}\left(\Omega_{m}\right)}<\infty
$$

As a consequence, the sequence $\left(u_{n}\right)_{n>1}$ is bounded in $H^{1}\left(\Omega_{m}\right)$. Due to the weak compactness of the unit ball of $H^{1}\left(\Omega_{m}\right)$, we can extract from $\left(u_{n}\right)_{n \geq 1}$ a subsequence that converges weakly to $u \in H^{1}\left(\Omega_{m}\right)$. According to Rellich's Theorem, $H^{1}\left(\Omega_{m}\right)$ is compactly embedded in $L^{2}\left(\Omega_{m}\right)$. We can therefore extract from the preceding subsequence a subsequence that converges strongly to $u$ in $L^{2}\left(\Omega_{m}\right)$, and also (up to one further extraction) that converges to $u$ almost everywhere in $\Omega_{m}$.

After applying a diagonal extraction procedure, we obtain a subsequence $\left(u_{n_{p}}\right)_{p \geq 1}$ of $\left(u_{n}\right)_{n \geq 1}$ and a function $u$ defined almost everywhere in $\Omega$ such that for each $m \geq 1, u_{n_{p}} \rightarrow u$ when $p \rightarrow+\infty$ weakly in $H^{1}\left(\Omega_{m}\right)$, strongly in $L^{2}\left(\Omega_{m}\right)$, and almost everywhere in $\Omega_{m}$. We have, for each $m \geq 1$, each $p \geq 1$,

$$
\int_{\Omega_{m}}\left|u_{n_{p}}\right|^{2} d x \leq \int_{\Omega}\left|u_{n_{p}}\right|^{2} d x=1
$$

and

$$
\int_{\Omega_{m}}\left|\left(-i \nabla-\mathbf{A}_{\boldsymbol{\alpha}}^{\mathbf{X}}\right) u_{n_{p}}\right|^{2} d x \leq 2 \sup _{q \geq 1} \lambda_{q}+2 \sup _{\Omega_{m}}\left|\mathbf{A}_{\boldsymbol{\alpha}}^{\mathbf{X}^{n}}-\mathbf{A}_{\boldsymbol{\alpha}}^{\mathbf{X}}\right|^{2} .
$$

Passing to the limit $p \rightarrow 0$, we get

$$
\int_{\Omega_{m}}|u|^{2} d x \leq 1
$$

and

$$
\int_{\Omega_{m}}\left|\left(-i \nabla-\mathbf{A}_{\boldsymbol{\alpha}}^{\mathbf{X}}\right) u\right|^{2} d x \leq 2 \sup _{q \geq 1} \lambda_{q} .
$$

We deduce that $u \in L^{2}(\Omega)$, with $\|u\|_{L^{2}(\Omega)} \leq 1$, and that $\left(-i \nabla-\mathbf{A}_{\boldsymbol{\alpha}}^{\mathbf{X}}\right) u$, as a distribution on $\Omega_{\mathbf{X}}$, belongs to $L^{2}(\Omega)$. 
For $m \geq 1$, let us note $\Gamma_{m}=\partial \Omega \cap \partial \Omega_{m}$. For $p \geq 1$, we have $\gamma_{0} u_{n_{p}}=0$ on $\Gamma_{m}$. Since $\left(u_{n_{p}}\right)_{p \geq 1}$ converges weakly to $u$ in $H^{1}\left(\Omega_{m}\right),\left(\gamma_{0} u_{n_{p}}\right)_{p \geq 1}$ converges weakly to $\gamma_{0} u$ in $L^{2}\left(\Gamma_{m}\right)$ and therefore $\gamma_{0} u=0$ on $\Gamma_{m}$. Since $\cup_{m=1}^{\infty} L^{2}\left(\Gamma_{m}\right)=L^{2}(\partial \Omega)$, we have shown $\gamma_{0} u=0$. According to Proposition 1.1. $u \in \mathcal{Q}_{\boldsymbol{\alpha}}^{\mathbf{X}}$.

Let us check that $u$ is a weak solution of the eigenvalue equation. Let $\varphi$ be a $C^{\infty}$-function with support in $\Omega_{\mathbf{X}}$. There exists $m \geq 1$ such that $\operatorname{supp}(\varphi) \subset \Omega_{m}$. For $p$ large enough,

$$
\int_{\Omega} u_{n_{p}} \overline{\left(-i \nabla-\mathbf{A}_{\boldsymbol{\alpha}}^{\mathbf{X}^{n_{p}}}\right)^{2} \varphi} d x=\lambda_{n_{p}} \int_{\Omega} u_{n_{p}} \bar{\varphi} d x
$$

Since $\left(-i \nabla-\mathbf{A}_{\boldsymbol{\alpha}}^{\mathbf{X}^{n p}}\right)^{2} \varphi$ converges uniformly on $\Omega$ to $\left(-i \nabla-\mathbf{A}_{\boldsymbol{\alpha}}^{\mathbf{X}}\right)^{2} \varphi$, we get,

$$
\int_{\Omega} u \overline{\left(-i \nabla-\mathbf{A}_{\boldsymbol{\alpha}}^{\mathbf{X}}\right)^{2} \varphi} d x=\lambda \int_{\Omega} u \bar{\varphi} d x
$$

and therefore

$$
\int_{\Omega}\left(-i \nabla-\mathbf{A}_{\boldsymbol{\alpha}}^{\mathbf{X}}\right) u \cdot \overline{\left(-i \nabla-\mathbf{A}_{\boldsymbol{\alpha}}^{\mathbf{X}}\right) \varphi} d x=\lambda \int_{\Omega} u \bar{\varphi} d x .
$$

By definition of $\mathcal{Q}_{\boldsymbol{\alpha}}^{\mathbf{X}}$, the preceding relation can be extended to any $\varphi \in \mathcal{Q}_{\boldsymbol{\alpha}}^{\mathbf{X}}$.

It remains to check that $u$ is not the trivial solution. To this end, we use the non-concentration property of Lemma 2.7 to show that $\left(u_{n_{p}}\right)_{p \geq 1}$ converges in $L^{2}(\Omega)$ to $u$. Indeed, if $\varepsilon>0$, there exists $m$ large enough so that, for all $p \geq 1$,

$$
\int_{S_{m}}\left|u_{n_{p}}(x)\right|^{2} d x \leq \frac{C N}{m}\left(\sup _{q \geq 1} \lambda_{q}+1\right) \leq \varepsilon,
$$

where $C$ is the constant appearing in Inequality $2.4, N$ is the number of balls composing $S_{m}$, and $1 / m$ the radius of these balls.

Let us pick such a $m$. Then

$$
\left\|u-u_{n_{p}}\right\|_{L^{2}(\Omega)} \leq\left\|u-u_{n_{p}}\right\|_{L^{2}\left(\Omega_{m}\right)}+\left\|u-u_{n_{p}}\right\|_{L^{2}\left(S_{m}\right)}
$$

so

$$
\left\|u-u_{n_{p}}\right\|_{L^{2}(\Omega)} \leq\left\|u-u_{n_{p}}\right\|_{L^{2}\left(\Omega_{m}\right)}+\|u\|_{L^{2}\left(S_{m}\right)}+\left\|u_{n_{p}}\right\|_{L^{2}\left(S_{m}\right)} .
$$

Hence we get

$$
\left\|u-u_{n_{p}}\right\|_{L^{2}(\Omega)} \leq\left\|u-u_{n_{p}}\right\|_{L^{2}\left(\Omega_{m}\right)}+2 \varepsilon .
$$

Since $\lim _{p \rightarrow \infty}\left\|u-u_{n_{p}}\right\|_{L^{2}\left(\Omega_{m}\right)}=0$, we have, for $p$ large enough,

$$
\left\|u-u_{n_{p}}\right\|_{L^{2}(\Omega)} \leq 3 \varepsilon \text {. }
$$

\subsection{End of the proof of the continuity}

We now prove Proposition 3.1, using the min-max characterization of the eigenvalues of $-\Delta_{\boldsymbol{\alpha}}^{\mathbf{X}}$. We still need a simple lemma.

Lemma 3.3. Let $\left(\mathbf{X}^{n}\right)_{n \geq 1}$ be such that $\mathbf{X}^{n} \rightarrow \mathbf{X}$ when $n \rightarrow \infty$. Then, for each $k \geq 1$,

$$
\limsup _{n \rightarrow \infty} \lambda_{k}\left(\mathbf{X}^{n}, \boldsymbol{\alpha}\right) \leq \lambda_{k}(\mathbf{X}, \alpha) .
$$


Proof. According to the Min-Max Formula, for any $\mathbf{Y} \in \mathbb{R}^{2 N}$,

$$
\lambda_{k}(\mathbf{Y}, \alpha)=\inf _{\varphi_{1}, \ldots, \varphi_{k} \in C_{c}^{\infty}\left(\Omega_{\mathbf{Y}}\right)} \max _{u \in \operatorname{vect}\left(\varphi_{1}, \ldots, \varphi_{k}\right)} \frac{\left\|\left(-i \nabla-\mathbf{A}_{\mathbf{\alpha}}^{\mathbf{Y}}\right) u\right\|^{2}}{\|u\|^{2}} .
$$

Let us pick $\varepsilon>0$. We fix a family $\varphi_{1}, \ldots, \varphi_{k} \in C_{c}^{\infty}\left(\Omega_{\mathbf{X}}\right)$ such that

$$
\max _{u \in \operatorname{vect}\left(\varphi_{1}, \ldots, \varphi_{k}\right)} \frac{\left\|\left(-i \nabla-\mathbf{A}_{\boldsymbol{\alpha}}^{\mathbf{X}}\right) u\right\|^{2}}{\|u\|^{2}} \leq \lambda_{k}(\mathbf{X}, \boldsymbol{\alpha})+\varepsilon .
$$

For every $n \geq 1$, large enough that $\operatorname{supp}\left(\varphi_{i}\right) \subset \Omega_{\mathbf{X}^{n}}$ for each $1 \leq i \leq k$, let us set

$$
\mu_{n}=\max _{u \in \operatorname{vect}\left(\varphi_{1}, \ldots, \varphi_{k}\right)} \frac{\left\|\left(-i \nabla-\mathbf{A}_{\boldsymbol{\alpha}}^{\mathbf{X}^{n}}\right) u\right\|^{2}}{\|u\|^{2}} .
$$

Let us fix $v_{n} \in \operatorname{vect}\left(\varphi_{1}, \ldots, \varphi_{k}\right)$ such that $\left\|v_{n}\right\|=1$ and $\left\|\left(-i \nabla-\mathbf{A}_{\boldsymbol{\alpha}}^{\mathbf{X}^{n}}\right) v_{n}\right\|^{2}=$ $\mu_{n}$. Let $\mu$ be a limit point of $\left(\mu_{n}\right)_{n \geq 1}$, with $\mu=\lim _{p \rightarrow \infty} \mu_{n_{p}}$. Since the space $\operatorname{vect}\left(\varphi_{1}, \ldots, \varphi_{k}\right)$ has finite dimension, we can, up to a subsequence, assume that there exists $v \in \operatorname{vect}\left(\varphi_{1}, \ldots, \varphi_{k}\right)$ such that $v_{n_{p}} \rightarrow v$ in $L^{2}(\Omega)$ when $p \rightarrow \infty$. Since the $\varphi_{i}$ 's are supported away from $\mathbf{X}$, it is easily seen that in that case

$$
\left(-i \nabla-\mathbf{A}_{\boldsymbol{\alpha}}^{\mathbf{X}^{n_{p}}}\right) v_{n_{p}} \rightarrow\left(-i \nabla-\mathbf{A}_{\boldsymbol{\alpha}}^{\mathbf{X}}\right) v
$$

in $L^{2}(\Omega)$ when $p \rightarrow \infty$.

Therefore

$$
\mu=\lim _{p \rightarrow \infty} \mu_{n_{p}}=\frac{\left\|\left(-i \nabla-\mathbf{A}_{\boldsymbol{\alpha}}^{\mathbf{X}}\right) v\right\|^{2}}{\|v\|^{2}} \leq \lambda_{k}(\mathbf{X}, \boldsymbol{\alpha})+\varepsilon .
$$

We have proved that

$$
\limsup _{n \rightarrow \infty} \mu_{n} \leq \lambda_{k}(\mathbf{X}, \boldsymbol{\alpha})+\varepsilon .
$$

Since $\lambda_{k}\left(\mathbf{X}^{n}, \boldsymbol{\alpha}\right) \leq \mu_{n}$ for all $n \geq 1$, we obtain

$$
\limsup _{n \rightarrow \infty} \lambda_{k}\left(\mathbf{X}^{n}, \boldsymbol{\alpha}\right) \leq \lambda_{k}(\mathbf{X}, \boldsymbol{\alpha})+\varepsilon .
$$

We get the desired result by letting $\varepsilon$ tend to 0 .

We now complete the proof of Proposition 3.1 by induction. Let us first consider the case $k=1$. According to Lemma 3.3,

$$
\limsup _{n \rightarrow \infty} \lambda_{1}\left(\mathbf{X}^{n}, \boldsymbol{\alpha}\right) \leq \lambda_{1}(\mathbf{X}, \boldsymbol{\alpha}) .
$$

The sequence $\left(\lambda_{1}\left(\mathbf{X}^{n}, \boldsymbol{\alpha}\right)\right)_{n \geq 1}$ is therefore bounded. According to Lemma 3.2 . its limit points are eigenvalues of $-\Delta_{\boldsymbol{\alpha}}^{\mathbf{X}}$. This implies

$$
\lambda_{1}(\mathbf{X}, \boldsymbol{\alpha}) \leq \liminf _{n \rightarrow \infty} \lambda_{1}\left(\mathbf{X}^{n}, \boldsymbol{\alpha}\right) .
$$

We conclude that

$$
\lim _{n \rightarrow \infty} \lambda_{1}\left(\mathbf{X}^{n}, \boldsymbol{\alpha}\right)=\lambda_{1}(\mathbf{X}, \boldsymbol{\alpha}) .
$$

Let $k \geq 2$. We assume that for $1 \leq j \leq k-1, \lim _{n \rightarrow \infty} \lambda_{j}\left(\mathbf{X}^{n}, \boldsymbol{\alpha}\right)=$ $\lambda_{j}(\mathbf{X}, \boldsymbol{\alpha})$. For each $n \geq 1,1 \leq j \leq k$, we fix $u_{n}^{j}$ an eigenfunction of $-\Delta_{\boldsymbol{\alpha}}^{\mathbf{X}^{n}}$ 
associated with $\lambda_{j}\left(\mathbf{X}^{n}, \boldsymbol{\alpha}\right)$ such that $\left\|u_{n}^{j}\right\|=1$. Lemma 3.2 tells us that, up to a subsequence, we can assume that for each $1 \leq j \leq k-1, u_{n}^{j} \rightarrow u^{j}$ when $n \rightarrow \infty$, where $u^{j}$ is an eigenfunction of $-\Delta_{\boldsymbol{\alpha}}^{\mathbf{X}}$ associated with the eigenvalue $\lambda_{j}(\mathbf{X}, \boldsymbol{\alpha})$. Now let us assume that $\lambda$ is a limit point for the sequence $\left(\lambda_{k}\left(\mathbf{X}^{n}, \boldsymbol{\alpha}\right)\right)_{n \geq 1}$, with $\lambda=\lim _{p \rightarrow \infty} \lambda_{k}\left(\mathbf{X}^{n_{p}}, \boldsymbol{\alpha}\right)$. Up to a subsequence, $u_{n_{p}}^{k} \rightarrow u^{k}$ in $L^{2}(\Omega)$ when $p \rightarrow$ $\infty$, where $u^{k}$ is an eigenfunction of $-\Delta_{\boldsymbol{\alpha}}^{\mathbf{X}}$ associated with $\lambda$ (always according to Lemma 3.2). For each $1 \leq j \leq k-1$, each $n \geq 1$, we have

$$
\left\langle u_{n_{p}}^{j}, u_{n_{p}}^{k}\right\rangle=0 .
$$

Passing to the limit in $L^{2}(\Omega)$, we get

$$
\left\langle u^{j}, u^{k}\right\rangle=0,
$$

and therefore

$$
\lambda_{k}(\mathbf{X}, \boldsymbol{\alpha}) \leq \lambda
$$

We have proved that

$$
\lambda_{k}(\mathbf{X}, \boldsymbol{\alpha}) \leq \liminf _{n \rightarrow \infty} \lambda_{k}\left(\mathbf{X}^{n}, \boldsymbol{\alpha}\right),
$$

which, together with Lemma 3.3 , gives

$$
\lim _{n \rightarrow \infty} \lambda_{k}\left(\mathbf{X}^{n}, \boldsymbol{\alpha}\right)=\lambda_{k}(\mathbf{X}, \boldsymbol{\alpha})
$$

\subsection{Some applications}

In the following, we fix $k \geq 1$. Our first result deals with one point moving toward the boundary of the domain. Let us assume that the set $\mathbb{R}^{2} \backslash \Omega$ can be written as the reunion of a finite number $K+1$ of closed and connected sets $D_{1}, D_{j}, \ldots, D_{K}$, with $D_{j}$ bounded for $1 \leq j \leq K$ and $D_{0}$ unbounded. Let us consider an Aharonov-Bohm operator with only one pole $X \in \mathbb{R}^{2}$. We have already noticed that the winding number of any path $\gamma$ contained in $\Omega$ is zero around any point in $D_{0}$ and, for each $1 \leq i \leq K$, is the same around all the points in $D_{i}$. According to Lemma 2.3, this implies that the function $X \mapsto \lambda_{k}(X, 1 / 2)$ is constant on each of the $D_{i}$. For each $1 \leq i \leq K$, let us note $\lambda_{k}^{i}(\Omega)$ the value of $\lambda_{k}(X, \alpha)$ for $X \in D_{i}$. Theorem 1.2 gives the following convergence result.

Corollary 3.4. Let $X$ be a point in $\partial \Omega$ and $\left(X^{n}\right)_{n \geq 1}$ be a sequence of points in $\Omega$ converging to $X$. Then,

1. if $X \in \partial D_{0}, \lim _{n \rightarrow \infty} \lambda_{k}\left(X^{n}, \alpha\right)=\lambda_{k}^{D}(\Omega)$,

2. if $X \in \partial D_{i}$ with $1 \leq i \leq K, \lim _{n \rightarrow \infty} \lambda_{k}\left(X^{n}, \alpha\right)=\lambda_{k}^{i}(\Omega)$.

Let us now consider an Aharonov-Bohm operator with two poles $X$ and $Y$, whose respective normalized flux are $\alpha$ and $\beta$. We restate Theorem 1.2 in that case.

Corollary 3.5. If $X \in \bar{\Omega}$, and $\left(X^{n}\right)_{n \geq 1},\left(Y^{n}\right)_{n \geq 1}$ are sequences of points in $\Omega$ converging to $X$, then,

$$
\lim _{n \rightarrow \infty} \lambda_{k}\left(\left(X^{n}, Y^{n}\right),(\alpha, \beta)\right)=\lambda_{k}(X, \alpha+\beta) .
$$

If $\alpha=\beta=1 / 2$, Lemma 2.3 implies $\lambda_{k}(X, \alpha+\beta)=\lambda_{k}^{D}(\Omega)$ 


\section{Analyticity with respect to the poles}

We now turn to the proof of Theorem 1.3 . Let us first give a brief outline. For $\mathbf{t}=\left(t_{1}, t_{2}, \ldots, t_{2 N-1}, t_{2 N}\right) \in \mathbb{R}^{2 N}$, we note $X_{1}(\mathbf{t})=X_{1}+t_{1} \mathbf{e}_{1}+$ $t_{2} \mathbf{e}_{2}, \ldots, X_{N}(\mathbf{t})=X_{N}+t_{2 N-1} \mathbf{e}_{1}+t_{2 N} \mathbf{e}_{2}$, and $\mathbf{X}(\mathbf{t})=\left(X_{1}(\mathbf{t}), \ldots, X_{N}(\mathbf{t})\right)$. We construct a suitable unitary mapping $U(\mathbf{t}): L^{2}(\Omega) \rightarrow L^{2}(\Omega)$ such that $q_{\boldsymbol{\alpha}}^{\mathbf{X}} \circ U(\mathbf{t})$ is an analytic family of type $(a)$ with respect to the variable $\mathbf{t}$ in the sense of Kato (cf [11, Chap. 7, Sec. 4]). The theorem is then a direct application of the Kato-Rellich regular perturbation theory.

Let us first construct $U(\mathbf{t})$. For $r>0$ and $1 \leq i \leq N$, we note $B_{i}=B\left(X_{i}, r\right)$ and $B_{i}^{\prime}=B\left(X_{i}, r / 2\right)$. We choose $r$ small enough so that $B_{i} \cap \partial \Omega=\emptyset$ and $B_{i} \cap B_{j}=\emptyset$ for $i \neq j$. Next we fix, for each $1 \leq i \leq N$, a smooth function $\chi_{i}$ satisfying

i. $0 \leq \chi_{i} \leq 1$

ii. $\chi_{i}=1$ on $B_{i}^{\prime}$;

iii. $\chi_{i}=0$ on $\mathbb{R}^{2} \backslash B_{i}$.

We then note

$$
\mathbf{V}_{\mathbf{t}}(x)=\sum_{i=1}^{N} \chi_{i}(x)\left(t_{2 i-1} \mathbf{e}_{1}+t_{2 i} \mathbf{e}_{2}\right)
$$

and

$$
\Phi_{\mathbf{t}}(x)=x+\mathbf{V}_{\mathbf{t}}(x) .
$$

Using the inverse function theorem and the mean value inequality, it is easy to check that, for $|\mathbf{t}|$ small enough, $\Phi_{\mathbf{t}}$ is a smooth diffeomorphism that sends $\Omega$ onto itself. Furthermore, we have $\Phi_{\mathbf{t}}\left(X_{i}\right)=X_{i}(\mathbf{t})$ for $1 \leq i \leq N$. We now define $U(\mathbf{t}): L^{2}(\Omega) \rightarrow L^{2}(\Omega)$ by

$$
U(\mathbf{t}) u=J\left(\left(\Phi_{\mathbf{t}}\right)^{-1}\right)^{1 / 2} u \circ\left(\Phi_{\mathbf{t}}\right)^{-1},
$$

with $J\left(\left(\Phi_{\mathbf{t}}\right)^{-1}\right)=\left|\operatorname{det}\left(D\left(\left(\Phi_{\mathbf{t}}\right)^{-1}\right)\right)\right|$.

The map $U(\mathbf{t})$ is unitary and satisfies $U(\mathbf{t})\left(C_{c}^{\infty}\left(\Omega_{\mathbf{X}}\right)\right)=C_{c}^{\infty}\left(\Omega_{\mathbf{X}(\mathbf{t})}\right)$.

For $u \in C_{c}^{\infty}\left(\Omega_{\mathbf{X}}\right)$, we define $r_{\mathbf{t}}(u)=q_{\boldsymbol{\alpha}}^{\mathbf{X}(\mathbf{t})}((U(\mathbf{t})) u)$. We note $\mathbf{A}=\mathbf{A}_{\boldsymbol{\alpha}}^{\mathbf{X}}$ and $\mathbf{A}_{\mathbf{t}}=\mathbf{A}_{\boldsymbol{\alpha}}^{\mathbf{X}(\mathbf{t})}$ for better readability. A straightforward computation gives the formula

$$
r_{\mathbf{t}}(u)=\int_{\Omega}\left|-i J\left(\Phi_{\mathbf{t}}\right)^{1 / 2}\left(D\left(\Phi_{\mathbf{t}}\right)^{T}\right)^{-1} \nabla\left(J\left(\Phi_{\mathbf{t}}\right)^{-1 / 2} u\right)-\mathbf{A}_{\mathbf{t}} \circ \Phi_{\mathbf{t}} u\right|^{2} d x .
$$

We can then write

$$
r_{\mathbf{t}}(u)=\int_{\Omega}\left|(-i \nabla-\mathbf{A}) u+E\left(\Phi_{\mathbf{t}}\right)(-i \nabla-\mathbf{A}) u+F\left(\Phi_{\mathbf{t}}\right) u\right|^{2} d x,
$$

with

$$
E\left(\Phi_{\mathbf{t}}\right)=\left(D\left(\Phi_{\mathbf{t}}\right)^{T}\right)^{-1}-I d
$$

and

$$
F\left(\Phi_{\mathbf{t}}\right)=\left(D\left(\Phi_{\mathbf{t}}\right)^{T}\right)^{-1} \mathbf{A}-\mathbf{A}_{\mathbf{t}} \circ \Phi_{\mathbf{t}}-i J\left(\Phi_{\mathbf{t}}\right)^{1 / 2}\left(D\left(\Phi_{\mathbf{t}}\right)^{T}\right)^{-1} \nabla\left(J\left(\Phi_{\mathbf{t}}\right)^{-1 / 2}\right) .
$$


Let us note that $\lim _{\mathbf{t} \rightarrow 0} \sup _{\Omega}\left|E\left(\Phi_{\mathbf{t}}\right)\right|=0$. Let us also note that $\left|F\left(\Phi_{\mathbf{t}}\right)\right|$ is uniformly bounded when $\mathbf{t}$ tends to 0 . The difficulties in the proof of this latter assertion only appear in the neighborhood of a pole $X_{i}$, where $\mathbf{A}$ has a singularity. Since the poles are far from each other, it is enough to prove the assertion in the case of one pole. We assume

$$
\mathbf{A}(x)=\alpha \frac{(x-X)^{\perp}}{|x-X|^{2}} .
$$

We note $B=B(X, r), B^{\prime}=B(X, r / 2)$. Let us note $v(\mathbf{t})=t_{1} \mathbf{e}_{1}+t_{2} \mathbf{e}_{2}$ for concision. If $x \in B^{\prime}, \Phi_{\mathbf{t}}(x)=x+v(\mathbf{t})$, and therefore

$F\left(\Phi_{\mathbf{t}}(x)\right)=\mathbf{A}(x)-\mathbf{A}_{\mathbf{t}}\left(\Phi_{\mathbf{t}}(x)\right)=\alpha\left(\frac{(x-X)^{\perp}}{|x-X|^{2}}-\frac{(x+v(\mathbf{t})-X-v(\mathbf{t}))^{\perp}}{|x+v(\mathbf{t})-X-v(\mathbf{t})|^{2}}\right)=0$.

If $x \notin B^{\prime}$, we have $|x-X| \geq r / 2$. We can chose $\mathbf{t}=\left(t_{1}, t_{2}\right)$ small enough so that, for all $y \in \mathbb{R}^{2},\left|\Phi_{\mathbf{t}}(y)-y\right| \leq r / 8$. In that case,

$$
\left|\Phi_{\mathbf{t}}(x)-\Phi_{\mathbf{t}}(X)\right| \geq|x-X|-\left|x-\Phi_{\mathbf{t}}(x)\right|-\left|\Phi_{\mathbf{t}}(X)-X\right| \geq \frac{r}{2}-\frac{r}{8}-\frac{r}{8}=\frac{r}{4} .
$$

This shows that both $|\mathbf{A}(x)|$ and $\left|\mathbf{A}_{\mathbf{t}}(x)\right|$ are bounded by $4|\alpha| / r$ for $\mathbf{t}$ small enough. Therefore, there exists a constant $C$, independent of $x$ and $\mathbf{t}$, such that $\left|F\left(\Phi_{\mathbf{t}}\right)(x)\right| \leq C$ for $\mathbf{t}$ small enough.

Expanding Equation 4.1, we find

$$
\begin{array}{r}
r_{\mathbf{t}}(u)=q_{\boldsymbol{\alpha}}^{\mathbf{X}}(u)+\int_{\Omega}\left|E\left(\Phi_{\mathbf{t}}\right)(-i \nabla-\mathbf{A}) u\right|^{2} d x+\int_{\Omega}\left|F\left(\Phi_{\mathbf{t}}\right) u\right|^{2} d x \\
+2 \operatorname{Re}\left(\int_{\Omega}(-i \nabla-\mathbf{A}) u^{T} E\left(\Phi_{\mathbf{t}}\right) \overline{(-i \nabla-\mathbf{A}) u} d x\right) \\
+2 \operatorname{Re}\left(\int_{\Omega}(-i \nabla-\mathbf{A}) u \cdot \overline{F\left(\Phi_{\mathbf{t}}\right) u} d x\right) \\
+2 \operatorname{Re}\left(\int_{\Omega} F\left(\Phi_{\mathbf{t}}\right) u^{T} E\left(\Phi_{\mathbf{t}}\right) \overline{(-i \nabla-\mathbf{A}) u} d x\right) .
\end{array}
$$

Applying the Cauchy-Schwarz and Young inequalities, we find

$$
\begin{aligned}
\left|r_{\mathbf{t}}(u)-q_{\boldsymbol{\alpha}} \mathbf{X}(u)\right| \leq\left(2 \sup _{\Omega}\left\|E\left(\Phi_{\mathbf{t}}\right)\right\|+2 \sup _{\Omega}\left\|E\left(\Phi_{\mathbf{t}}\right)\right\|^{2}+\frac{1}{2}\right) q_{\boldsymbol{\alpha}}^{\mathbf{X}}(u) & \\
& +4 \sup _{\Omega}\left|F\left(\Phi_{\mathbf{t}}\right)\right|^{2}\|u\|_{L^{2}(\Omega)}^{2} .
\end{aligned}
$$

We conclude that there exist $0<a<1$ and $b \geq 0$, independent of $u$, such that, for $\mathbf{t}$ small enough,

$$
\left|r_{\mathbf{t}}(u)-q_{\boldsymbol{\alpha}}^{\mathbf{X}}(u)\right| \leq a q_{\boldsymbol{\alpha}}^{\mathbf{X}}(u)+b\|u\|_{L^{2}(\Omega)}^{2} .
$$

The family of forms $\mathbf{t} \rightarrow r_{\mathbf{t}}$ is therefore uniformly $q_{\boldsymbol{\alpha}}^{\mathbf{X}}$-bounded for $\mathbf{t}$ in a neighborhood of 0 . According to standard results on the perturbation of sesquilinear sectorial forms (c.f. [11, Th. VI-1.33]), this means that the form domain of $r_{\mathbf{t}}(u)$ is equal to $\mathcal{Q}_{\boldsymbol{\alpha}}^{\mathbf{X}}$ for all $\mathbf{t}$ small enough. It is obvious that for $u \in \mathcal{Q}_{\boldsymbol{\alpha}}^{\mathbf{X}}, r_{\mathbf{t}}(u)$ depends analytically on $\mathbf{t}$. The family $\mathbf{t} \rightarrow r_{\mathbf{t}}$ therefore depends analytically on 
$\mathbf{t}$ in the sense of Kato. Theorem 1.3 is a consequence of the general perturbation theory.

Let us now give a proof of Theorem 1.4 . We first give a precise definition of a $K \mathrm{X}$-real eigenfunction, as announced in the introduction. We assume in the rest of this section that $\alpha_{i} \in \mathbb{Z}+1 / 2$ for all $1 \leq i \leq N$. According to Lemma 2.3. there exists a gauge function $\psi$ defined on $\Omega_{\mathbf{X}}$ such that $-i \bar{\psi} \nabla \psi=2 \mathbf{A}_{\boldsymbol{\alpha}}^{\mathbf{X}}$. Let us fix such a $\psi$. We define $K_{\mathbf{X}}$ by

$$
K_{\mathbf{X}} u=\psi \bar{u},
$$

and say that a function $u$ is $K_{\mathbf{X}}$-real if $K_{\mathbf{X}} u=u$.

A direct calculation shows that

$$
-\Delta_{\boldsymbol{\alpha}}^{\mathbf{X}} \circ K_{\mathbf{X}}=K_{\mathbf{X}} \circ\left(-\Delta_{\boldsymbol{\alpha}}^{\mathbf{X}}\right) .
$$

We can therefore choose a basis of $K_{\mathbf{X}}$-real eigenfunctions for $-\Delta_{\boldsymbol{\alpha}}^{\mathbf{X}}$. We will use the local description of the nodal lines of a $K_{\mathbf{X}}$-real eigenfunction of $-\Delta_{\boldsymbol{\alpha}}^{\mathbf{X}}$ given in [2] (see also [15]).

Theorem 4.1. Let $\boldsymbol{\alpha} \in(\mathbb{Z}+1 / 2)^{N}$ and $X \in \mathbb{R}^{2 N}$. Let us fix $1 \leq i \leq N$ with $X_{i} \in \Omega$. Let $k \geq 1$ and assume that $u$ is a $K_{\mathbf{X}}$-real eigenfunction associated with $\lambda_{k}(\mathbf{X}, \boldsymbol{\alpha})$. Let $(r, \theta)$ be the polar coordinates centered at $X_{i}$ in a neighborhood of $X_{i}$. There exist a non-negative integer $m$ and $C^{1}$ functions $f$ and $g$ such that

$$
\begin{gathered}
f(0) \neq 0, \\
u(x)=r^{m+1 / 2} f(x),
\end{gathered}
$$

and

$$
\left(-i \nabla-\mathbf{A}_{\boldsymbol{\alpha}}^{\mathbf{X}}(x)\right) u(x)=r^{m-1 / 2} g(x) .
$$

Furthermore, $2 m+1$ is the number of nodal lines meeting at $X_{i}$.

The basic idea of our proof of Theorem 1.4 is the following. We construct a family of diffeomorphisms $\Phi_{h}$ that depends on a parameter $h>0$. Using the Feynman-Hellmann formula, we compute $\lambda_{k}^{\prime}(0)$ (which does not depend on $h$ ) as an integral $I(h)$ depending on $h$. We then use Theorem 4.1, with $m \geq 1$, to show that $\lim _{h \rightarrow 0} I(h)=0$.

To simplify notation, let us assume $X_{i}=0$. Let us first construct a family of diffeomorphisms $\Phi_{h, t}$ that allows us to write the eigenvalue problem on the fixed domain $\Omega_{\mathbf{X}}$. We fix $r>0$ such that $B(0, r) \subset \Omega$ and $X_{j} \notin B(0, r)$ for $j \neq i$. We then fix a vector field $\mathbf{V} \in C^{\infty}\left(\mathbb{R}^{2}, \mathbb{R}^{2}\right)$ such that

1. $\mathbf{V}(x)=v$ if $x \in B(0, r / 2)$,

2. $\mathbf{V}(x)=0$ if $x \notin B(0, r)$,

We then define, for $t>0, \Phi_{h, t}(x)=x+t \mathbf{V}\left(h^{-1} x\right)$, and $\mathbf{X}(t)=\Phi_{h, t}(\mathbf{X})$. It is easy to see that for any fixed $h>0$, for $|t|$ small enough, $\Phi_{h, t}$ is a family of diffeomorphisms such that $\Phi_{h, t}(\Omega)=\Omega$. As in the proof of Theorem 1.3 , we define the family of unitary operators $U(h, t): L^{2}(\Omega) \rightarrow L^{2}(\Omega)$ by

$$
U(h, t) u=J\left(\Phi_{h, t}^{-1}\right)^{1 / 2} u \circ \Phi_{h, t}^{-1},
$$


and the family of sesquilinear forms $r_{h, t}=q_{\boldsymbol{\alpha}}^{\mathbf{X}(t)} \circ U(h, t)$. The same reasoning as in the proof of Theorem 1.3 shows that $t \mapsto r_{h, t}$ is analytic in the sense of Kato in a neighborhood of 0 . We can therefore apply the Feynman-Hellmann formula:

$$
\lambda_{k}^{\prime}(0)=\partial_{t} r_{h, t}(u)_{\mid t=0},
$$

where $u$ is an eigenfunction associated with $\lambda_{k}(0)$ such that $\|u\|_{L^{2}(\Omega)}=1$. We set $\mathbf{A}=\mathbf{A}_{\boldsymbol{\alpha}}^{\mathbf{X}}, \mathbf{A}_{t}=\mathbf{A}_{\boldsymbol{\alpha}}^{\mathbf{X}(t)}$, and keep the notation used in the proof of Theorem 1.3. We have

$$
\begin{aligned}
& r_{h, t}(u)=q_{\boldsymbol{\alpha}}^{\mathbf{X}}(u)+ \int_{\Omega}\left|E\left(\Phi_{h, t}\right)(-i \nabla-\mathbf{A}) u\right|^{2} d x+\int_{\Omega}\left|F\left(\Phi_{t}\right) u\right|^{2} d x \\
&+2 \operatorname{Re}\left(\int_{\Omega}(-i \nabla-\mathbf{A}) u^{T} E\left(\Phi_{h, t}\right) \overline{(-i \nabla-\mathbf{A}) u} d x\right) \\
&+2 \operatorname{Re}\left(\int_{\Omega}(-i \nabla-\mathbf{A}) u \cdot \overline{F\left(\Phi_{h, t}\right) u} d x\right) \\
&+2 \operatorname{Re}\left(\int_{\Omega} F\left(\Phi_{h, t}\right) u^{T} E\left(\Phi_{h, t}\right) \overline{(-i \nabla-\mathbf{A}) u} d x\right) .
\end{aligned}
$$

We apply Equation 4.2 to the preceding expression.

Since $\Phi_{h, t}(x)=x+t \mathbf{V}\left(\frac{x}{h}\right)$, we find

$$
D \Phi_{h, t}(x)=I d+\frac{t}{h} D \mathbf{V}\left(\frac{x}{h}\right) .
$$

We deduce

$$
\partial_{t} E\left(\Phi_{h, t}\right)_{\mid t=0}(x)=\partial_{t}\left(\left(D\left(\Phi_{h, t}\right)^{T}\right)^{-1}\right)_{\mid t=0}(x)=-\frac{1}{h}(D \mathbf{V})^{T}\left(\frac{x}{h}\right) .
$$

We also have

$$
J\left(D \Phi_{h, t}\right)(x)=\operatorname{det}\left(I d+\frac{t}{h} D \mathbf{V}\left(\frac{x}{h}\right)\right)
$$

which gives

$$
\partial_{t}\left(J\left(D \Phi_{h, t}\right)(x)\right)_{\mid t=0}=\frac{1}{h} \operatorname{Tr}(D \mathbf{V})\left(\frac{x}{h}\right) .
$$

From this we deduce

$$
\begin{array}{r}
\partial_{t}\left(\nabla_{x}\left(J\left(D \Phi_{h, t}\right)^{-1 / 2}\right)\left(\frac{x}{h}\right)\right)_{\mid t=0}=\nabla_{x}\left(\partial_{t}\left(J\left(D \Phi_{h, t}\right)^{-1 / 2}\right)\left(\frac{x}{h}\right)_{\mid t=0}\right) \\
=\nabla_{x}\left(-\frac{1}{2 h} \operatorname{Tr}(D \mathbf{V})\left(\frac{x}{h}\right)\right)=-\frac{1}{2 h^{2}} \nabla_{x} \operatorname{Tr}(D \mathbf{V})\left(\frac{x}{h}\right)
\end{array}
$$

We obtain

$\partial_{t} F\left(\Phi_{h, t}\right)(x)_{\mid t=0}=\partial_{t}\left(\left(D\left(\Phi_{h, t}\right)^{T}\right)^{-1} \mathbf{A}(x)-\mathbf{A}_{t} \circ \Phi_{h, t}(x)\right)_{\mid t=0}-\frac{i}{2 h^{2}} \nabla_{x} \operatorname{Tr}(D \mathbf{V})\left(\frac{x}{h}\right)$.

For $|x| \leq h r / 2$, we have $\Phi_{h, t}(x)=x+v$. We therefore have

$$
\left(D\left(\Phi_{\mathbf{t}}\right)^{T}\right)^{-1} \mathbf{A}(x)-\mathbf{A}_{t} \circ \Phi_{h, t}(x)=0
$$


and

$$
\partial_{t}\left(\left(D\left(\Phi_{\mathbf{t}}\right)^{T}\right)^{-1} \mathbf{A}(x)-\mathbf{A}_{t} \circ \Phi_{h, t}(x)\right)_{\mid t=0}=0 .
$$

We now do the computations for $|x|>h r / 2$. We write

$$
\mathbf{A}=\alpha_{i} \frac{x^{\perp}}{|x|^{2}}+\mathbf{A}^{\prime},
$$

where $\mathbf{A}$ is smooth on a neighborhood of 0 .

We have

$$
\partial_{t}\left(\frac{(x+t \mathbf{V}(x / h))^{\perp}}{|x+t \mathbf{V}(x / h)|^{2}}\right)_{\mid t=0}=\frac{1}{|x|^{2}}-\frac{x \cdot \mathbf{V}(x / h)}{|x|^{4}} x^{\perp} .
$$

We conclude that there exists a constant $C$, such that for all $h>0$ and all $x \in \Omega$,

$$
\left|\partial_{t} E\left(\Phi_{h, t}\right)(x)_{\mid t=0}\right| \leq \frac{C}{h}
$$

and

$$
\left|\partial_{t} F\left(\Phi_{h, t}\right)(x)_{\mid t=0}\right| \leq \frac{C}{h^{2}} .
$$

We have

$$
\begin{aligned}
\partial_{t} r_{h, t}(u)_{\mid t=0}=2 \operatorname{Re}\left(\int_{\Omega}(-\right. & \left.i \nabla-\mathbf{A}) u^{T} \partial_{t} E\left(\Phi_{h, t}\right)_{\mid t=0} \overline{(-i \nabla-\mathbf{A}) u} d x\right) \\
& +2 \operatorname{Re}\left(\int_{\Omega} \partial_{t} F\left(\Phi_{h, t}\right)_{\mid t=0} u \cdot \overline{(-i \nabla-\mathbf{A}) u} d x\right) .
\end{aligned}
$$

We deduce immediately

$$
\begin{aligned}
\left|\partial_{t} r_{h, t}(u)_{\mid t=0}\right| \leq \frac{2 C}{h} \|(-i \nabla & -\mathbf{A}) u \|_{L^{2}(B(0, h r))}^{2} \\
& +\frac{2 C}{h^{2}}\|(-i \nabla-\mathbf{A}) u\|_{L^{2}(B(0, h r)}\|u\|_{L^{2}(B(0, h r))} .
\end{aligned}
$$

According to Theorem 4.1, there exist an integer $m \geq 0$ and $C^{1}$-functions $f$ and $g$ such that in a neighborhood of 0 ,

$$
u(x)=|x|^{m+1 / 2} f(x)
$$

and

$$
(-i \nabla-\mathbf{A}) u=|x|^{m-1 / 2} g(x),
$$

where $f(0) \neq 0$ and $2 m+1$ is the number of nodal lines meeting at 0 .

From this we deduce the estimates

$$
\begin{gathered}
\int_{B(0, h r)}|u|^{2} d x=O\left(h^{2 m+3}\right), \\
\int_{B(0, h r)}|(-i \nabla-\mathbf{A}) u|^{2} d x=O\left(h^{2 m+1}\right),
\end{gathered}
$$

and finally

$$
\left|\partial_{t} r_{h, t}(u)_{\mid t=0}\right|=O\left(h^{2 m}\right) .
$$


Since we have assumed that at least three nodal lines meet at 0 , we have in fact $m \geq 1$ and we deduce

$$
\left|\partial_{t} r_{h, t}(u)_{\mid t=0}\right|=O\left(h^{2}\right) .
$$

Since this holds for all $h>0$ and since the left-hand side of Equation 4.2 does not depend on $h$, we obtain

$$
\lambda_{k}^{\prime}(0)=0,
$$

which concludes the proof.

The Kato-Rellich perturbation theory gives some information in the case where $\lambda_{k}(\mathbf{X}, \boldsymbol{\alpha})$ is not simple. Let us denote by $d$ the multiplicity of $\lambda_{k}(\mathbf{X}, \boldsymbol{\alpha})$. Let us fix an $N$-tuple $\left(\mathbf{v}_{1}, \ldots, \mathbf{v}_{N}\right)$ of vectors in $\mathbb{R}^{2}$. For any $t$ in $\mathbb{R}$, we note

$$
\mathbf{X}(t)=\left(X_{1}+t \mathbf{v}_{1}, \ldots, X_{N}+t \mathbf{v}_{N}\right) .
$$

There exist $d$ functions $t \mapsto \lambda_{1}(t), \ldots, t \mapsto \lambda_{d}(t)$ (not necessarily distinct), analytical in a neighborhood of 0 , such that for $1 \leq j \leq d, \lambda_{j}(0)=\lambda_{k}(\mathbf{X}, \boldsymbol{\alpha})$ and $\lambda_{j}(t)$ is an eigenvalue of the operator $-\Delta_{\boldsymbol{\alpha}}^{\mathbf{X}(t)}$. There also exist $d$ analytical functions $t \mapsto u_{j}(t)$ from a neighborhood of 0 to $L^{2}(\Omega)$ such that $\left\|u_{j}(t)\right\|_{L^{2}(\Omega)}=$ 1 and $u_{j}(t)$ is an eigenfunction associated with $\lambda_{j}(t)$. They can be chosen so that $u_{j}(t)$ is $K_{\mathbf{X}}$-real.

Furthermore, the Feynman-Hellmann formula then tells us that

$$
\lambda_{j}^{\prime}(0)=\left\langle\partial_{t}\left(-\Delta_{\boldsymbol{\alpha}}^{\mathbf{X}(t)}\right)_{\mid t=0} u_{j}(0), u_{j}(0)\right\rangle .
$$

Let us now fix $1 \leq i_{0} \leq N$ and consider the case where $\mathbf{v}_{i}=0$ for all $i \neq i_{0}$ (we only move the point $X_{i_{0}}$ ) and $\alpha_{i_{0}} \in \mathbb{Z}+1 / 2$. Assume that there exists $1 \leq j_{0} \leq d$ such that $u_{j_{0}}(0)$ is $K_{\mathbf{X}}$-real and has at least three nodal lines meeting at $X_{i}$. The proof of Theorem 1.4 applies; we obtain $\lambda_{j_{0}}^{\prime}(0)=0$. However, the existence of a $K_{\mathbf{X}}$-real eigenfunction associated with $\lambda_{k}(\mathbf{X}, \boldsymbol{\alpha})$ with at least three nodal lines meeting at $X_{i_{0}}$ is a priori not enough to ensure the existence of $1 \leq j_{0} \leq d$ such that $\lambda_{j_{0}}^{\prime}(0)=0$.

\section{Minimal partitions}

\subsection{Magnetic characterization and critical points}

One motivation for this work was the investigation of the properties of minimal partitions. A $k$-partition $\mathcal{P}$ of $\Omega$, with $k$ a positive integer, is a set $\mathcal{P}=\left\{D_{1}, \ldots, D_{k}\right\}$ of $k$ disjoint, connected, and open sets contained in $\Omega$. It is said to be minimal if the quantity

$$
\max _{1 \leq i \leq k} \lambda_{1}^{D}\left(D_{i}\right)
$$

is minimal among all the $k$-partitions.

This problem has recently been the subject of various studies. In particular, the paper [9], by B. Helffer, T. Hoffmann-Ostenhof, and S. Terracini, shows that a minimal partition of $\Omega$ exists for any integer $k$ provided $\partial \Omega$ is sufficiently regular. It also proves regularity properties of minimal partitions. The paper [8] by B. Helffer and T. Hoffmann-Ostenhof, establishes a connection between 
minimal partitions and nodal partitions for an Aharonov-Bohm operator. We can summarize it in the following statement. We recall that a nodal domain of a $K_{\mathbf{X}}$-real eigenfunction $u$ of $-\Delta_{\boldsymbol{\alpha}}^{\mathbf{X}}$ is a connected component of the set $\Omega \backslash u^{-1}(\{0\})$.

Theorem 5.1. Let us assume that $\Omega$ is a connected (possibly with holes) regular open set. Let $k \geq 1$ be an integer and $\mathcal{P}=\left\{D_{1}, \ldots, D_{k}\right\}$ be a minimal $k$ partition. There exist a finite number of points $X_{1}, \ldots, X_{N}$ in $\mathbb{R}^{2}$ such that $\mathcal{P}$ is the nodal partition (i.e. the set of all nodal domains) of a $K_{\mathbf{X}}$-real eigenfunction $u$ of $-\Delta_{\boldsymbol{\alpha}}^{\mathbf{X}}$, with

$$
\begin{aligned}
\text { and } & \mathbf{X}=\left(X_{1}, \ldots, X_{N}\right) \\
& \boldsymbol{\alpha}=(1 / 2, \ldots, 1 / 2) .
\end{aligned}
$$

Furthermore, $u$ is associated with the eigenvalue $\lambda_{k}(\mathbf{X}, \boldsymbol{\alpha})$.

Roughly speaking, this result shows that a minimal partition is nodal, provided that we add enough poles with a normalized flux of $1 / 2$. Let us note that we may have to add poles both in $\Omega$ and in $\mathbb{R}^{2} \backslash \bar{\Omega}$. One can indeed define the boundary of a sufficiently regular partition (which can be informally described has the union of the boundaries of all the $D_{i}$ 's), and it has almost the same properties as the nodal set of a real eigenfunction of $-\Delta_{\Omega}^{D}$. However, there may be singular points where an odd number of lines meet. We have to add a pole at these points. In the case where $\Omega$ is not simply connected, we may also have to add a pole in some holes (i.e. in some bounded connected components of $\left.\mathbb{R}^{2} \backslash \bar{\Omega}\right)$.

Theorem 5.1 allows us to give a spectral characterization of minimal partitions. Let us note

$$
\mathfrak{L}_{k}(\Omega)=\inf \left\{\max _{1 \leq i \leq k} \lambda_{i}\left(D_{i}\right) ;\left\{D_{1}, \ldots, D_{k}\right\} k \text {-partition of } \Omega\right\} .
$$

If $\mathbf{X} \in \mathbb{R}^{2 N}$, with distinct $X_{i}$ 's, and $\boldsymbol{\alpha}=(1 / 2, \ldots, 1 / 2)$, we denote by $L_{k}(\mathbf{X}, \boldsymbol{\alpha})$ the smallest eigenvalue of $-\Delta_{\boldsymbol{\alpha}}^{\mathbf{X}}$ that has a $K_{\mathbf{X}}$-real eigenfunction with $k$ nodal domains. If there is no such eigenfunction, we set $L_{k}(\mathbf{X}, \boldsymbol{\alpha})=+\infty$. Then

$$
\mathfrak{L}_{k}(\Omega)=\inf _{N \geq 1} \inf _{\mathbf{X} \in \mathbb{R}^{2 N}} L_{k}(\mathbf{X}, \boldsymbol{\alpha}) .
$$

We can combine Theorem 5.1 with the results of Section 4 to show that $\mathfrak{L}_{k}(\Omega)$ is a critical value for the $k$-th eigenvalue of an Aharonov-Bohm operator. Let us give a precise statement.

Theorem 5.2. Let us assume that $\Omega$ is a connected open set, $k$ a positive integer, and $\mathcal{P}$ a minimal $k$-partition of $\Omega$. We denote by $\mathbf{X}=\left(X_{1}, \ldots, X_{N}\right)$ and $\boldsymbol{\alpha}=(1 / 2 \ldots, 1 / 2)$ the poles and fluxes defined in Theorem 5.1. Let us additionally assume that the eigenvalue $\lambda_{k}(\mathbf{X}, \boldsymbol{\alpha})$ is simple. The point $\mathbf{X}$ is then critical for the function $\mathbf{Y} \mapsto \lambda_{k}(\mathbf{Y}, \boldsymbol{\alpha})$, which is defined and analytic in a neighborhood of $\mathbf{X}$.

Proof. According to Theorem 1.3. $\mathbf{Y} \mapsto \lambda_{k}(\mathbf{Y}, \boldsymbol{\alpha})$ is analytic in a neighborhood of $\mathbf{X}$. As allowed by Theorem 5.1, $\mathbf{X}$ has been chosen so that there exists a $K_{\mathbf{X}}$-real eigenfunction $u$ whose nodal partition is $\mathcal{P}$. To show that $\mathbf{X}$ is critical, it is enough to show that the gradient with respect to each point $X_{i}$, with $i \in\{1, \ldots, N\}$, is zero. There are two possible cases. 
- $X_{i} \in \mathbb{R}^{2} \backslash \bar{\Omega}$. In that case $\lambda_{k}(\mathbf{Y}, \boldsymbol{\alpha})=\lambda_{k}(\mathbf{X}, \boldsymbol{\alpha})$ if $\mathbf{Y}=\left(Y_{1}, \ldots, Y_{N}\right)$ is such that $Y_{j}=X_{j}$ for $j \neq i$ and $Y_{i}$ is in the same connected component of $\mathbb{R}^{2} \backslash \bar{\Omega}$ as $X_{i}$ (This is a consequence of Lemma 2.3.

- $X_{i} \in \Omega$. In that case at least three nodal lines of $u$ meet at $X_{i}$. Therefore, according to Theorem 1.4, $X_{i}$ is a critical point for the function

$$
Y \mapsto \lambda_{k}\left(\left(X_{1}, \ldots, X_{i-1}, Y, X_{i+1}, \ldots, X_{N}\right), \boldsymbol{\alpha}\right)
$$

The fact that $\mathbf{X}$ is a critical point is proved in the case $N=1$ for the minimal 3 -partitions of a simply connected domain in [6]. As far as we know, our result is new for a general $k$-partition.

\subsection{Domain with an axis of symmetry}

According to Theorem 5.1, minimal partitions of an open set are nodal partitions for an Aharonov-Bohm operator. This can help us to find good candidates to be minimal partitions of a given set. Let us explain a method that was used in the papers [4, 3, 5]. The authors of these papers where looking for minimal 3 -partitions of rectangles and circular sectors, but we will explain the method in more general terms.

Let $\Omega \in \mathbb{R}^{2}$ be a simply connected open set with a regular boundary. Let us also assume that the line $\left\{x_{2}=0\right\}$ is an axis of symmetry for $\Omega$. It seems reasonable to look for minimal partitions that have only one singular point in their boundary and that admit $\left\{x_{2}=0\right\}$ as an axis of symmetry. We therefore look for $X=(x, 0)$, a pole belonging to $\left\{x_{2}=0\right\}$, such that $\lambda_{3}(X, 1 / 2)$ admits a $K_{\mathbf{X}}$-real eigenfunction with three nodal domains.

In the case that we consider, there exists a convenient reformulation of the eigenvalue problem. We note $\Omega^{+}=\Omega \cap\left\{x_{2}>0\right\}, \Gamma^{+}=\partial \Omega \cap\left\{x_{2}>0\right\}$, and $\Omega \cap\left\{x_{2}=0\right\}=(A, B)$, with $A=(a, 0)$ and $B=(b, 0)$. We then define two eigenvalue problems for the Laplace operator in $\Omega^{+}$with mixed boundary condition.

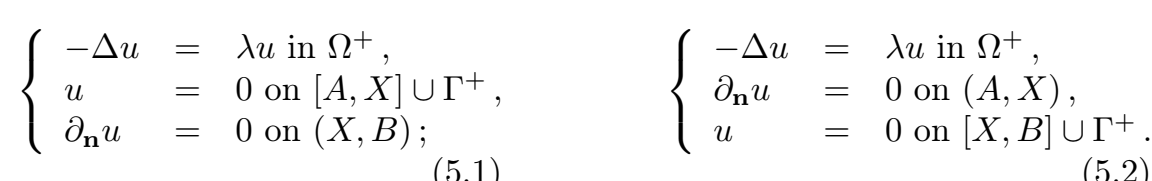

We denote by $\left(\lambda_{k}^{D N}(x)\right)_{k>1}$ (resp. $\left.\left(\lambda_{k}^{N D}(x)\right)_{k>1}\right)$ the eigenvalues of Problem (5.1) (resp. (5.2) ), counted with multiplicity. The following result is proved in 4.

Proposition 5.3. The spectrum of $-\Delta_{1 / 2}^{X}$ is the reunion (counted with multiplicities) of the sequences $\left(\lambda_{k}^{D N}(x)\right)_{k>1}$ and $\left(\lambda_{k}^{N D}(x)\right)_{k>1}$. Moreover, if $u$ is a real eigenfunction associated with $\lambda_{k}^{D \bar{N}}(x)$ (resp. $\lambda_{k}^{N D}(\bar{x})$ ), the symmetrization of $\mathcal{N}(u)$ with respect to $\left\{x_{2}=0\right\}$ is the nodal set of a $K_{\mathbf{X}}$-real eigenfunction of $-\Delta_{1 / 2}^{X}$ associated with $\lambda_{k}^{D N}(x)$ (resp. $\left.\lambda_{k}^{N D}(x)\right)$.

Proposition 5.3 motivates the study of Problems (5.1) and 5.2 . More precisely, the authors of [3, 5] compute, by a finite element method, $\lambda_{2}^{N D}(x)$ and the 
nodal lines of an associated eigenfunction $u$ for different values of the parameter $x \in(a, b)$. They look for a value $x_{0}$ of $x$ such that there is an eigenfunction $u$ associated with $\lambda_{2}^{N D}\left(x_{0}\right)$ that has one nodal line, which connects a point of $\Gamma^{+}$ with $X_{0}=\left(x_{0}, 0\right)$. In that case, the symmetrization of the nodal partition of $u$ gives a 3 -partition of $\Omega$ which is a plausible candidate to be minimal. The search for $x_{0}$ can be guided by the following result.

Proposition 5.4. Let $k$ be a positive integer. Let us assume that $x_{0} \in(a, b)$ is such that the eigenvalue $\lambda_{k}^{N D}\left(x_{0}\right)$ has a real eigenfunction with one nodal line hitting $\left\{x_{2}=0\right\}$ at $X_{0}=\left(x_{0}, 0\right)$. Let us additionally assume that $\lambda_{k}^{N D}\left(x_{0}\right)$ is a simple eigenvalue of $-\Delta_{1 / 2}^{X_{0}}$. Then $x_{0}$ is a point of inflexion for the function $x \mapsto \lambda_{k}^{N D}(x)$.

Proof. Let us first note that according to Proposition 5.3 and since $\lambda_{k}^{N D}\left(x_{0}\right)$ is a simple eigenvalue of $-\Delta_{1 / 2}^{X_{0}}$, there is a positive integer $\ell$ such that $\lambda_{k}^{N D}(x)=$ $\lambda_{\ell}(X, 1 / 2)$, with $X=(x, 0)$, for $x$ in a neighborhood of $x_{0}$. According to Theorem 1.3 the function $x \mapsto \lambda_{k}^{N D}(x)$ is therefore analytic in a neighborhood of $x_{0}$. The hypothesis on the nodal line, together with Proposition 5.3, implies that there is a $K_{\mathbf{X}}$-real eigenfunction associated with $\lambda_{\ell}\left(X_{0}, 1 / 2\right)$ that has at least three nodal lines meeting at $X_{0}$. According to Theorem 1.4 this implies $\left(\lambda_{k}^{N D}\right)^{\prime}\left(x_{0}\right)=0$. But, as seen on Problem 5.2 the function $x \mapsto \lambda_{k}^{N D}(x)$ is non-increasing. This implies $\left(\lambda_{k}^{N D}\right)^{\prime \prime}\left(x_{0}\right)=0$.

There exists of course a similar result for $x \mapsto \lambda_{k}^{D N}(x)$. The following example illustrates this proposition when looking for a 3-partition of a circular sector of radius 1 and angular opening $\pi / 3{ }^{1}$. Figure 1 shows a point of inflexion $x_{0} \simeq 0.64$ of the function $x \mapsto \lambda_{2}^{N D}(x)$. Figure 2 shows the 3 -partition obtained after symmetrization of the nodal partition of an eigenfunction associated with $\lambda_{2}^{N D}\left(x_{0}\right)$.

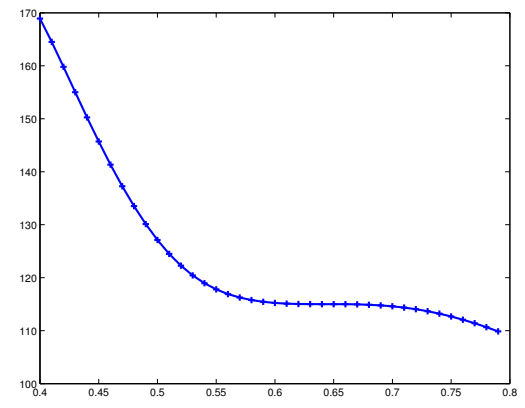

Figure 1: Eigenvalue $\lambda_{2}^{N D}(x)$ as a function of $x$.

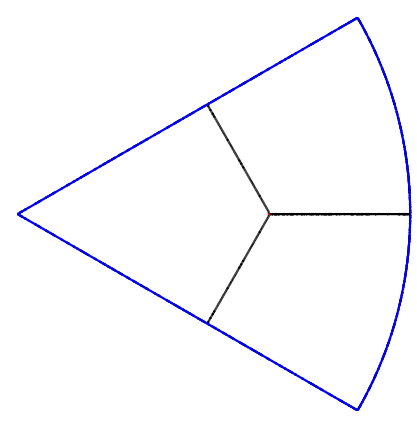

Figure 2: Nodal set of a third eigenfunction of an Aharonov-Bohm operator.

Let us note that the point of inflexion in Figure 1 is hard to determine precisely in practice. We only obtain an approximate value. To draw Figure 2 , we look for $x_{0}$ around this approximate value by computing the nodal set for different values of $x$.

\footnotetext{
${ }^{1}$ We thank V. Bonnaillie-Noël for the computations and the figures.
} 


\section{References}

[1] Y. Aharonov and D. Bohm. Significance of electromagnetic potentials in the quantum theory. Phys. Rev., 115:485-491, Aug 1959.

[2] B. Alziary, J. Fleckinger-Pellé, and P. Takáč. Eigenfunctions and Hardy inequalities for a magnetic Schrödinger operator in $\mathbb{R}^{2}$. Math. Methods Appl. Sci., 26(13):1093-1136, 2003.

[3] V. Bonnaillie-Noël and B. Helffer. Numerical analysis of nodal sets for eigenvalues of Aharonov-Bohm Hamiltonians on the square with application to minimal partitions. Exp. Math., 20(3):304-322, 2011.

[4] V. Bonnaillie-Noël, B. Helffer, and T. Hoffmann-Ostenhof. AharonovBohm Hamiltonians, isospectrality and minimal partitions. J. Phys. A, 42(18):185203, 20, 2009.

[5] V. Bonnaillie-Noël and C. Léna. Spectral minimal partitions of a sector. Discrete Contin. Dyn. Syst. Ser. B, 19(1):27-53, 2014.

[6] V. Bonnaillie-Noël, B. Noris, M. Nys, and S. Terracini. On the eigenvalues of Aharonov-Bohm operators with varying poles. ArXiv e-prints, October 2013.

[7] B. Helffer, M. Hoffmann-Ostenhof, T. Hoffmann-Ostenhof, and M. P. Owen. Nodal sets for groundstates of Schrödinger operators with zero magnetic field in non-simply connected domains. Comm. Math. Phys., 202(3):629-649, 1999 .

[8] B. Helffer and T. Hoffmann-Ostenhof. On a magnetic characterization of spectral minimal partitions. J. Eur. Math. Soc. (JEMS), 15(6):2081-2092, 2013.

[9] B. Helffer, T. Hoffmann-Ostenhof, and S. Terracini. Nodal domains and spectral minimal partitions. Ann. Inst. H. Poincaré Anal. Non Linéaire, 26(1):101-138, 2009.

[10] L. Hillairet and C. Judge. The eigenvalues of the Laplacian on domains with small slits. Trans. Amer. Math. Soc., 362(12):6231-6259, 2010.

[11] T. Kato. Perturbation Theory for Linear Operators. Springer-Verlag, Berlin, second edition, 1976.

[12] A. Laptev and T. Weidl. Hardy inequalities for magnetic Dirichlet forms. In Mathematical results in quantum mechanics (Prague, 1998), pages 299305. Birkhäuser, Basel, 1999.

[13] H. Leinfelder. Gauge invariance of Schrödinger operators and related spectral properties. J. Operator Theory, 9(1):163-179, 1983.

[14] M. Melgaard, E.-M. Ouhabaz, and G. Rozenblum. Negative discrete spectrum of perturbed multivortex Aharonov-Bohm Hamiltonians. Ann. Henri Poincaré, 5(5):979-1012, 2004. 
[15] B. Noris and S. Terracini. Nodal sets of magnetic Schrödinger operators of Aharonov-Bohm type and energy minimizing partitions. Indiana Univ. Math. J., 59(4):1361-1403, 2010.

[16] M. Reed and B. Simon. Methods of Modern Mathematical physics. II. Fourier Analysis, Self-Adjointness. Academic Press, New York, 1975. 